\title{
Optimization of thin noise barrier designs using Evolutionary Algorithms and a Dual BEM Formulation*
}

\author{
R. Toledo, J. J. Aznárez, O. Maeso, D. Greiner \\ Instituto Universitario de Sistemas Inteligentes y Aplicaciones Numéricas en Ingeniería \\ (SIANI) Universidad de Las Palmas de Gran Canaria \\ Edificio Central del Parque Científico y Tecnológico \\ Campus Universitario de Tafira, 35017, Las Palmas de Gran Canaria, Spain \\ \{rtoledo, jjaznarez, omaeso, dgreiner\}@siani.es, web: http://www.siani.es
}

April 2014

\begin{abstract}
This work aims at assessing the acoustic efficiency of different thin noise barrier models. These designs frequently feature complex profiles and their implementation in shape optimization processes may not always be easy in terms of determining their topological feasibility. A methodology to conduct both overall shape and top edge optimisations of thin cross section acoustic barriers by idealizing them as profiles with null boundary thickness is proposed. This procedure is based on the maximization of the insertion loss of candidate profiles proposed by an evolutionary algorithm. The special nature of these sort of barriers makes necessary the implementation of a complementary formulation to the classical Boundary Element Method (BEM). Numerical simulations of the barriers performance are conducted by use of a 2D Dual BEM code in eight different barrier configurations (covering overall shaped and top edge configurations; spline curved and polynomial shaped based designs; rigid and noise absorbing boundaries materials). Results obtained show the usefulness of representing complex thin barrier configurations as null boundary thickness-like models.
\end{abstract}

Keywords: Thin noise barriers, Shape optimisation, Genetic Algorithms, Dual Boundary Element Formulation

\section{Introduction}

The inclusion of sound barriers for abating the negative effects of road traffic noise near residential areas is a broadly used strategy. Considerable research work and studies focused on sound diffraction around barriers have been carried out in the past two decades, specifically in the prediction of the performance and the development of more efficient designs. Among all of the different numerical methods available concerning the issue, the Boundary Element Method (BEM hereinafter) is one of

${ }^{*}$ This is the pre-peer reviewed version of the following article: Toledo R, Aznarez JJ, Maeso O, Greiner D. Optimization of thin noise barrier designs using evolutionary algorithms and a Dual BEM formulation. J Sound Vib 2015;334:219-38, which has been published in final form at http://dx.doi.org/10.1016/j.jsv.2014.08.032 
the broadly used. Remarkable work using the singular BEM for assessing the acoustic efficiency of sound noise barriers have been carried out to date. In 1980, Seznec [1 implements this methodology to assess the diffracted sound field behind a barrier. Hothersall et al. [2, 3] make use of this technique to study the performance of a vertical screen and compare it with different types of barriers with diffusive elements on their top. Watts and Morgan 4 predict the acoustic behaviour of a sound-interferencetype device added on the top of an existing straight barrier, yielding a significant improvement in the screening performance. Crombie et al. 5] study the performance of multiple-edge barriers, concluding that the addition of side-panels leads to a significant increase in acoustic efficiency over a simple vertical screen. Monazzam and Lam [6] carry out a comparison study between noise barriers with quadratic residue diffuser (QRD) tops and different top-edge profiles barriers, for both rigid and with absorptive coverage. In the same line, Ishizuka and Fujiwara [7] conclude that providing the top of noise barriers with soft edges significantly improves their efficiency. Configuration modifications provide only a slight improvement, though. Okubo and Fujiwara [8] assess the acoustic efficiency of the so called waterwheel cylinder installed on the top-edge of noise barriers to produce an approximate soft surface (a surface with a null sound pressure level), concluding that these designs are strongly frequency dependent. Jean et al. [9] study the influence of both source and ground type in the assessment of the efficiency of a straight, a T-shaped and a cylindrical top barrier. To supplement this compendium, other notable work for assessing the acoustic efficiency of noise barriers conducted by Maeso and Aznárez can be consulted in [10].

From a broader point of view, to the authors' knowledge, there are some noteworthy work involving the coupled use of BEM in outdoor acoustics in the literature. In this line, Tadeu et al. [11] propose a coupled BEM-TBEM formulation to model the propagation of sound in the presence of very thin elements. de Lacerda et al. [12] propose a 2D Dual BEM formulation for the treatment of non-thickness configurations and applied it to the assessment of a vertical and a T-shaped noise barrier modelled as thin bodies over an absorbing ground. Chen et al. [13] make use of a Dual BEM formulation to suppress the fictitious frequencies that arise when handling with non-thin elements.

In particular to the concerning issue here presented, the combined used of BEM and evolutionary algorithms has been used for shape design optimization in outdoor acoustics problems. Duhamel [14] starts off with a rectangular volumetric structure built of equally-sized bricks to lead to the final optimised shapes with non-inner holes and fillings. Baulac et al. [15] assess the performance of Tshaped barriers with different series of wells covered with a reactive surface on the top. Greiner et al. [16, 17] conduct the study of a single- and a multi-objective design optimization of a Y-shaped noise barrier; the consideration of uncertainties in the optimum design have also been handled in [18. Grubeša et al. [19] carry out a 3D optimization of both acoustic performance and economical feasibility of a noise barrier built from different modules with varying cross-sections. A more recent research, also covers the inclusion of an innovization procedure for multiobjective noise barrier optimum design in Deb et al. [20].

In this line, a procedure for the shape design optimisation of noise barriers by coupling BEM with an evolutionary algorithm is conducted in this paper. Two-dimensional sound propagation problems concerning an infinite, coherent mono-frequency source of sound, placed parallel to an infinite noise barrier that stands on a flat plane (ground) of uniform admittance are studied. The sound propagation analysis is performed in the frequency domain. Expression of the fitness function to be maximized throughout the shape optimisation process is written in terms of this response. 
The principal novelty of this work lies in the fact that the proposed Dual BEM formulation is applied in the study of noise barriers featured with very thin boundaries, idealized as null boundary thickness-like models. This simplification of reality greatly facilitates the geometric definition of barrier profiles, having no major influence on the acoustic performance [12]. The special nature of these type of barriers makes every node of the discretization hold both the pressure and the flux value at each side of it, i.e., $2 n$ unknowns per $n$ nodes. The inclusion of an additional BEM formulation (hyper-singular) combined with the classical one (singular) provides a compatible system of equations that allows the problem to be solved. The coupling of an evolutionary algorithm with the Dual BEM code allows to obtain interesting acoustic solutions avoiding the complexity associated with the geometric generation of volumetric structures.

To the authors' knowledge, the procedure described in this paper is the first joint implementation of evolutionary algorithms and a Dual BEM formulation concerning this issue. Fig. 1 shows the usefulness of representing complex volumetric structures as null boundary thickness-like models. The procedure for the geometric definition of the studied noise barriers, the fundamental aspects of the Dual BEM formulation implemented and the main features of the evolutionary algorithm software used are thoroughly and clearly explained in the next sections. As application, eight different barrier configurations of practical interest in the topic here presented, proposing more efficient designs in each case, are assessed. These profiles cover a wide range of designs, from complex straight boundary configurations to curve-shaped profiles. The paper is structured as follows: in section 2, the modelling and discretization by implementation of a Dual BEM formulation is explained. Section 3 deals with the noise barrier design problem definition and section 4 relates the shape optimisation formulation. Section 5 follows with the application of the proposed methodology to the assessment of the acoustic efficiency of different barrier designs. Finally, section 6 shows results and discussion, and section 7 covers the conclusions of the paper.

\section{Modelling and discretization by implementing a Dual BEM for- mulation}

The next lines are focused on the description of the implemented Dual BEM formulation to make possible the numerical treatment of thin noise barriers idealized as null boundary thickness profiles (see Fig. 11). The special nature of these type of barriers makes necessary the addition of a complementary formulation (hyper-singular) that coupled with the conventional BEM formulation yields a compatible system of equations.

\subsection{Singular BEM formulation}

The integral equation for the $i$ boundary point to be solved by the singular BEM formulation can be expressed as follows:

$$
c_{i} p_{i}+f_{\Gamma_{b}} p \frac{\partial p^{*}}{\partial \mathbf{n}_{j}} d \Gamma=p_{0}^{*}+\int_{\Gamma_{b}} \frac{\partial p}{\partial \mathbf{n}_{j}} p^{*} d \Gamma
$$

This integral equality just involves the boundary of the barrier under investigation. The $f$ symbol represents the integral along the boundary to be understood in the Cauchy principal value sense, once 
(a)

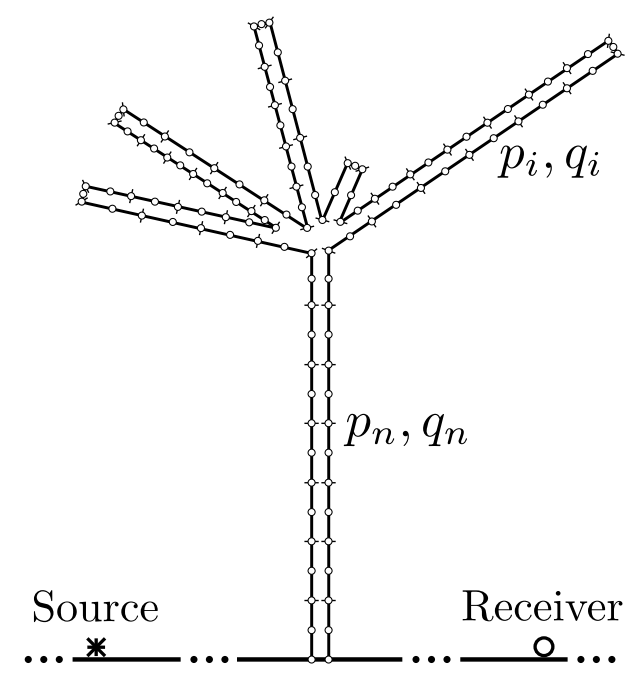

(b)

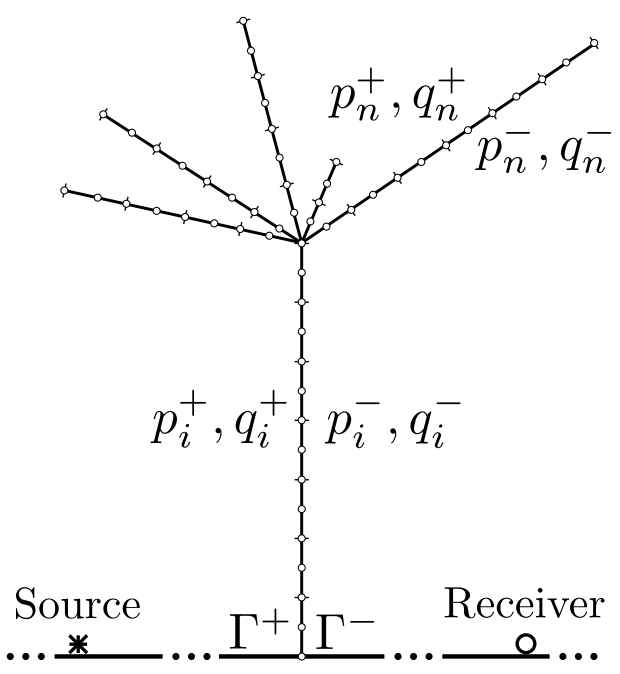

Figure 1: (a) Generic thin barrier modelled as a volumetric structure. (b) Idealization of the former barrier as a null boundary thickness profile.

the singularity around the collocation point $i$ has been extracted $\left(c_{i}\right)$. In (1), $p$ is the acoustic pressure field over the barrier surface and $p^{*}$ is the half-space fundamental solution (the acoustic pressure field when the source is placed at the collocation point $i$ over a plane with admittance $\beta_{g}$ (ground admittance) ) and $c_{i}$ is the free term. As a general rule: $c_{i}=\theta / 2 \pi$, where $\theta$ represents the inner angle to the boundary measured in radians. It is easily shown that $c_{i}=0.5$ for smooth boundaries.

The expressions of the fundamental solution and its derivative for a perfectly reflecting ground $\left(\beta_{g}=0\right)$ for bi-dimensional, harmonious problems are:

$$
\begin{aligned}
p^{*}(k, r) & =\frac{1}{2 \pi}\left[K_{0}(i k r)+K_{0}(i k \bar{r})\right] \\
\frac{\partial p^{*}}{\partial \mathbf{n}_{j}} & =-\frac{i k}{2 \pi}\left[K_{1}(i k r) \frac{\partial r}{\partial \mathbf{n}_{j}}+K_{1}(i k \bar{r}) \frac{\partial \bar{r}}{\partial \mathbf{n}_{j}}\right]
\end{aligned}
$$

being $i$ the imaginary unit, $k$ the wave number, and $r, \bar{r}$ the distances to the observation point from the collocation point and its symmetric point with respect to the ground plane, respectively. $K_{0}$ and $K_{1}$ are the Bessel modified functions of order 0 and 1 , respectively.

The application of (11) on each $i$ node of the boundary discretization leads to the following system of equations:

$$
\left(\mathbf{C}^{s}+\mathbf{H}\right) \cdot \mathbf{P}=\mathbf{G} \cdot \mathbf{Q}+\mathbf{P}_{0}^{*}
$$

where $\mathbf{C}^{s}$ is a diagonal matrix whose entries involve the free term values at the nodes of the discretization, $\mathbf{P}, \mathbf{Q}$ are the pressure and the flux (the derivative of the pressure with respect to the normal at 
each boundary node) vectors, $\mathbf{P}_{\mathbf{0}}^{*}$ vector stores the values of the fundamental solution concerning the external noise source, and $\mathbf{H}, \mathbf{G}$ are matrices whose entries are associated with the integration cores of the singular BEM formulation involving just the variables of the problem along the barrier boundary:

$$
h_{k}^{i j}=\int_{\Gamma_{j}} \frac{\partial p^{*}}{\partial \mathbf{n}_{j}} \phi_{k} d \Gamma_{j} \quad ; \quad g_{k}^{i j}=\int_{\Gamma_{j}} p^{*} \phi_{k} d \Gamma_{j}
$$

with $i$ being the collocation point, $j$ the observation point and $\phi_{k}$ the shape functions with quadratic approximation of the local variable $\xi$ along the element under integration.

\subsection{Hyper-singular BEM formulation}

The integral equation for the $i$ boundary point to be solved by the hyper-singular BEM formulation can be written as follows:

$$
c_{i}\left(\frac{\partial p_{i}}{\partial \mathbf{n}_{i}}\right)+f_{\Gamma_{b}} p \frac{\partial^{2} p^{*}}{\partial \mathbf{n}_{i} \partial \mathbf{n}_{j}} d \Gamma_{b}=f_{\Gamma_{b}} \frac{\partial p^{*}}{\partial \mathbf{n}_{i}} \frac{\partial p}{\partial \mathbf{n}_{j}} d \Gamma_{b}+\frac{\partial p_{0}^{*}}{\partial \mathbf{n}_{i}}
$$

where the $f$ and $f$ symbols represent the integral along the boundary to be understood in the Hadamard finite part integral and in the Cauchy principal value sense, respectively. As the Hölder condition must be satisfied at the collocation point $i$, hyper-singular formulation of the method demands the source placement to be inside the element (non-nodal collocation). Therefore, $c_{i}=0.5$ in (5) in all situations.

Expression (6) shows the values of the derivatives of the fundamental solution implied in the hyper-singular integral equation (5):

$$
\begin{aligned}
\frac{\partial p^{*}}{\partial \mathbf{n}_{i}}=-\frac{i k}{2 \pi} & {\left[K_{1}(i k r) \frac{\partial r}{\partial \mathbf{n}_{i}}+K_{1}(i k \bar{r}) \frac{\partial \bar{r}}{\partial \mathbf{n}_{I}}\right] } \\
\frac{\partial^{2} p^{*}}{\partial \mathbf{n}_{i} \partial \mathbf{n}_{j}}=\frac{(i k)^{2}}{2 \pi} & {\left[\left(K_{2}(i k r) \frac{\partial r}{\partial \mathbf{n}_{i}} \frac{\partial r}{\partial \mathbf{n}_{j}}+\frac{1}{r} K_{1}(i k r) \mathbf{n}_{i} \cdot \mathbf{n}_{j}\right)+\right.} \\
& \left.\left(K_{2}(i k \bar{r}) \frac{\partial \bar{r}}{\partial \mathbf{n}_{I}} \frac{\partial \bar{r}}{\partial \mathbf{n}_{j}}+\frac{1}{\bar{r}} K_{1}(i k \bar{r}) \mathbf{n}_{I} \cdot \mathbf{n}_{j}\right)\right]
\end{aligned}
$$

As in (1), $i$ is the imaginary unit, $k$ the wave number and $r, \bar{r}$ the distances to the observation point from the collocation point and its symmetric point with respect to the ground plane, respectively. It is worth making a distinction here regarding the normal vectors involved in the expressions above. $\mathbf{n}_{j}$ is the normal to the boundary at the integration point and $\mathbf{n}_{i}\left(n_{x}^{i}, n_{y}^{i}\right), \mathbf{n}_{I}\left(n_{x}^{i},-n_{y}^{i}\right)$ represent the normal vectors to the real boundary at the collocation point $(i)$ and at its symmetric point $(I)$ placed on a fictitious, symmetric boundary with respect to the ground plane, respectively. $K_{1}$ and $K_{2}$ represent the Bessel modified functions of order 1 and 2, respectively.

The application of (5) on each $i$ node of the boundary discretization leads to the following system of equations:

$$
\mathbf{M} \cdot \mathbf{P}=\left(\mathbf{L}-\mathbf{C}^{h}\right) \cdot \mathbf{Q}+\mathbf{Q}_{0}^{*}
$$


where $\mathbf{C}^{h}$ is a is a diagonal matrix with entry values of $0.5, \mathbf{P}, \mathbf{Q}$ are the pressure and the flux (the derivative of the pressure with respect to normal at each boundary node) vectors, $\mathbf{Q}_{0}^{*}$ array stores the values of the derivative of the fundamental solution concerning the external noise source, and $\mathbf{M}, \mathbf{L}$ are matrices whose entries are associated with the integration cores of the hyper-singular BEM formulation involving just the variables of the problem along the barrier boundary:

$$
m_{k}^{i j}=\int_{\Gamma_{j}} \frac{\partial^{2} p^{*}}{\partial \mathbf{n}_{i} \mathbf{n}_{j}} \phi_{k} d \Gamma_{j} \quad ; \quad l_{k}^{i j}=\int_{\Gamma_{j}} \frac{\partial p^{*}}{\partial \mathbf{n}_{i}} \phi_{k} d \Gamma_{j}
$$

The numerical strategies employed in the evaluation of both the singular and the hyper-singular BEM intregrals have been developed and implemented in a computer code by following the patterns proposed by Sáez et al. [21].

\subsection{Dual BEM formulation}

Fig. 2(a) represents a generic thin-cross section noise barrier to be solved by the Dual BEM formulation. After a discretization process, each node holds the values of pressure and flux with respect to the boundary normal $\left(p^{+}, q^{+}, p^{-}, q^{-}\right.$hereinafter $)$.

(a)

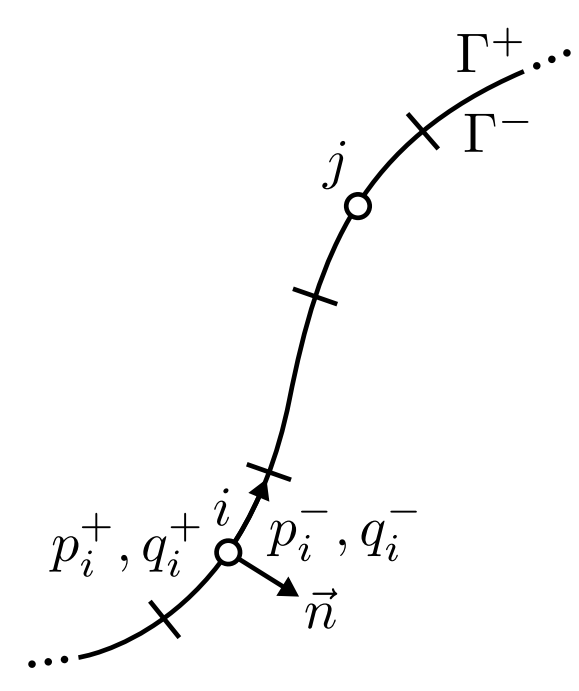

(b)

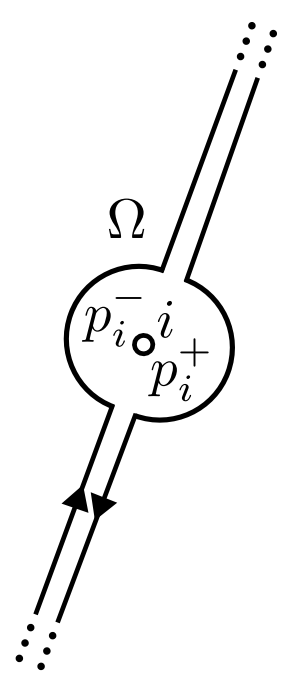

Figure 2: (a) Idealization of a generic thin-cross section noise barrier profile as null thickness boundaries. (b) Strategy used to avoid the singularity around the collocation point in BEM formulation.

Fig. 2(b) represents the strategy used to isolate the singularity of the method in this type of domains. Thus, the matrix equality of the singular BEM formulation for thin-cross section noise 
barriers can be expressed as follows:

$$
c_{i}\left(p_{i}^{+}+p_{i}^{-}\right)+\sum_{j=1}^{N}\left(H_{j}^{+} p_{j}^{+}+H_{j}^{-} p_{j}^{-}\right)=\sum_{j=1}^{N}\left(G_{j}^{+} q_{j}^{+}+G_{j}^{-} q_{j}^{-}\right)+p_{0}^{*}
$$

being $N$ the overall nodes number of the discretization over the boundary. Taking into account that $\mathbf{n}^{+}=-\mathbf{n}^{-}$, it is easily shown that:

$$
H_{j}^{+}=-H_{j}^{-} \quad ; \quad G_{j}^{+}=G_{j}^{-}
$$

Following the mathematical notation used by de Lacerda et al. [22], the final expression can be written for the sake of clarity as follows:

$$
c_{i} \Sigma p_{i}+\sum_{j=1}^{N} H_{j}^{+} \Delta p_{j}=\sum_{j=1}^{N} G_{j}^{+} \Sigma q_{j}+p_{0}^{*}
$$

where:

$$
\Sigma p_{i}=p_{i}^{+}+p_{i}^{-} ; \Delta p_{j}=p_{j}^{+}-p_{j}^{-} ; \Sigma q_{j}=q_{j}^{+}+q_{j}^{-}
$$

For smooth boundaries $c_{i}=0.5$ in (11). Furthermore, considering that these type of profiles demand a non-nodal collocation at unbound extremes of boundaries, the free term is equally assumed as 0.5 in such cases.

According to Fig. 2, the hyper-singular expression concerning these type of geometries can be written as follows:

$$
\left(\frac{1}{2}\right)\left(\frac{\partial p_{i}^{+}}{\partial \mathbf{n}_{i}^{+}}+\frac{\partial p_{i}^{-}}{\partial \mathbf{n}_{i}^{+}}\right)+\sum_{j=1}^{N}\left(M_{j}^{+} p_{j}^{+}+M_{j}^{-} p_{j}^{-}\right)=\sum_{j=1}^{N}\left(L_{j}^{+} q_{j}^{+}+L_{j}^{-} q_{j}^{-}\right)
$$

where:

$$
\frac{\partial p_{i}^{-}}{\partial \mathbf{n}_{i}^{+}}=-q_{i} \quad ; \quad M_{j}^{+}=-M_{j}^{-} \quad ; \quad L_{j}^{+}=L_{j}^{-}
$$

The hyper-singular formulation of the method requires the collocation point $j$ to be inside the element. In this way, the final expression can be expressed as follows:

$$
\left(\frac{1}{2}\right) \Delta q_{i}+\sum_{j=1}^{N} M_{j}^{+} \Delta p_{j}=\sum_{j=1}^{N} L_{j}^{+} \Sigma q_{j}+q_{0}^{*}
$$

The absorptive capacity of the barrier boundary is usually determined by means of the Robin boundary condition, so the pressure value and its derivative at each node are related:

$$
q_{j}^{+}=-i k \beta_{\Gamma}^{+} p_{j}^{+} \quad ; \quad q_{j}^{-}=-i k \beta_{\Gamma}^{-} p_{j}^{-}
$$


with $\beta_{\Gamma}$ being a complex value (based on the empiric relation proposed by Delany and Bazley [23]) that represents the admittance of the boundary $\Gamma$ for a particular frequency. In this way, the following can be written:

$$
\begin{aligned}
& \Delta q_{j}=\mathrm{A}^{-} \Sigma p_{j}+\mathrm{A}^{+} \Delta p_{j} \\
& \Sigma q_{j}=\mathrm{A}^{+} \Sigma p_{j}+\mathrm{A}^{-} \Delta p_{j}
\end{aligned}
$$

being:

$$
\mathrm{A}^{+}=-\frac{i k}{2}\left(\beta_{\Gamma}^{+}+\beta_{\Gamma}^{-}\right) \quad ; \quad \mathrm{A}^{-}=-\frac{i k}{2}\left(\beta_{\Gamma}^{+}-\beta_{\Gamma}^{-}\right)
$$

Substituting (17) into (11) and (15) the following system of equations is obtained:

$$
\left[\begin{array}{cc}
\frac{\mathbf{I}}{2}-\mathbf{G}^{+} \mathrm{A}^{+} & \mathbf{H}^{+}-\mathbf{G}^{+} \mathrm{A}^{-} \\
\frac{\mathrm{A}^{-}}{2} \mathbf{I}_{-} \mathbf{L}^{+} \mathrm{A}^{+} & \frac{\mathrm{A}^{+}}{2} \mathbf{I}+\mathbf{M}^{+}-\mathbf{L}^{+} \mathrm{A}^{-}
\end{array}\right]\left[\begin{array}{c}
\Sigma \mathbf{P} \\
\Delta \mathbf{P}
\end{array}\right]=\left[\begin{array}{c}
\mathbf{P}_{0}^{*} \\
\mathbf{Q}_{0}^{*}
\end{array}\right]
$$

with $\mathbf{I}$ being the identity matrix.

The matrix system above represents the final Dual BEM expressions for thin cross section barriers. For cases in which the boundaries are perfectly rigid $\left(\beta_{\Gamma}^{+}=\beta_{\Gamma}^{-}=0\right)$ the variables of the problem uncouple and $\Delta p_{j}$ is then directly obtained, resulting in faster computational times with respect to other cases.

Once the variables of the problems are known, their corresponding values at any point of the domain can be easily obtained by applying (20).

$$
p_{i}=p_{0}^{*}+\left(\sum_{j=1}^{N} G_{j}^{+} \Sigma q_{j}-\sum_{j=1}^{N} H_{j}^{+} \Delta p_{j}\right)
$$

\subsection{Discretization}

The Dual BEM code in this paper uses quadratic elements with three nodal points both to get the acoustic pressure level along the boundary (21) and to fit the barrier profile. This discretization process is frequency-dependent (with four elements per wavelength, at least).

$$
p^{i}=\phi_{1} p_{1}^{i}+\phi_{2} p_{2}^{i}+\phi_{3} p_{3}^{i}
$$

being:

$$
\phi_{1}=\frac{\xi}{2}(\xi-1) ; \phi_{2}=1-\xi^{2} ; \phi_{3}=\frac{\xi}{2}(\xi+1)
$$

where $\xi$ represents the local coordinate within the element with side limits $(-1,1)$ (see Fig. 3).

Fig. 3 represents the strategy used in the code for the hyper-singular BEM formulation. As previously mentioned, a non-nodal collocation is required in the extreme nodes of the elements. Extensive 


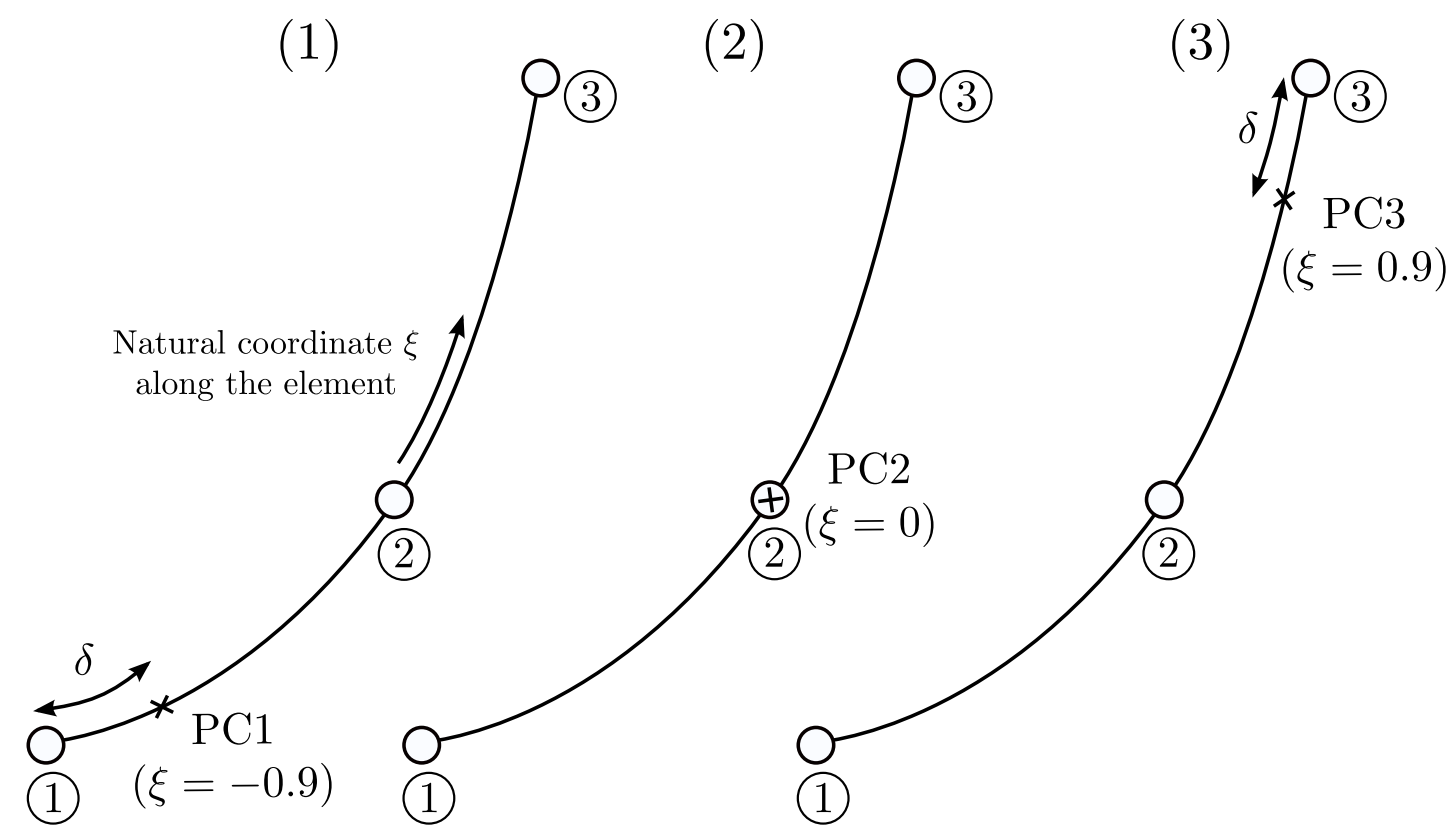

Figure 3: Non nodal collocation points at the bound limits of the element when dealing with the hypersingular BEM formulation [21]: (1) collocation point PC1; (2) collocation point PC2; (3) collocation point PC3.

references concerning the choice of $\delta$ value can be found in scientific literature. For the cases here presented, the reallocation of such nodes has been carried out with a well-proven distance of $\delta=5 \%$ of the element length for the point displacement towards inside. Differences in results associated with the election of this strategy are negligible, particularly given the fact that sound pressure levels of interest here are those neither at the barrier boundaries nor at the barrier corners but at the receiver points.

As for the singular BEM formulation, the code here presented makes use of nodal collocation with the exception of the nodes placed at non-connected extremes of boundaries, where a non-nodal collocation strategy is employed.

Some special procedures to tackle both sharp-angled boundaries and sharp angles between boundaries are considered. In order to assure the convergence of the numerical integrations of nearly singular integrals, the computing code used in this work implements two strategies. One of them is based on the procedure proposed by Telles [24], consisting in the reallocation and concentration of the Gauss points around the point with the minimum $r$ distance within the element under integration. The other strategy consists in the subdivision of the associated element from the barrier discretization into multiple subintervals, depending on the minimum $r$ distance to the cuasi-singular point. The final result is the overall sum of the numerical integration applied to each subinterval of the element. 


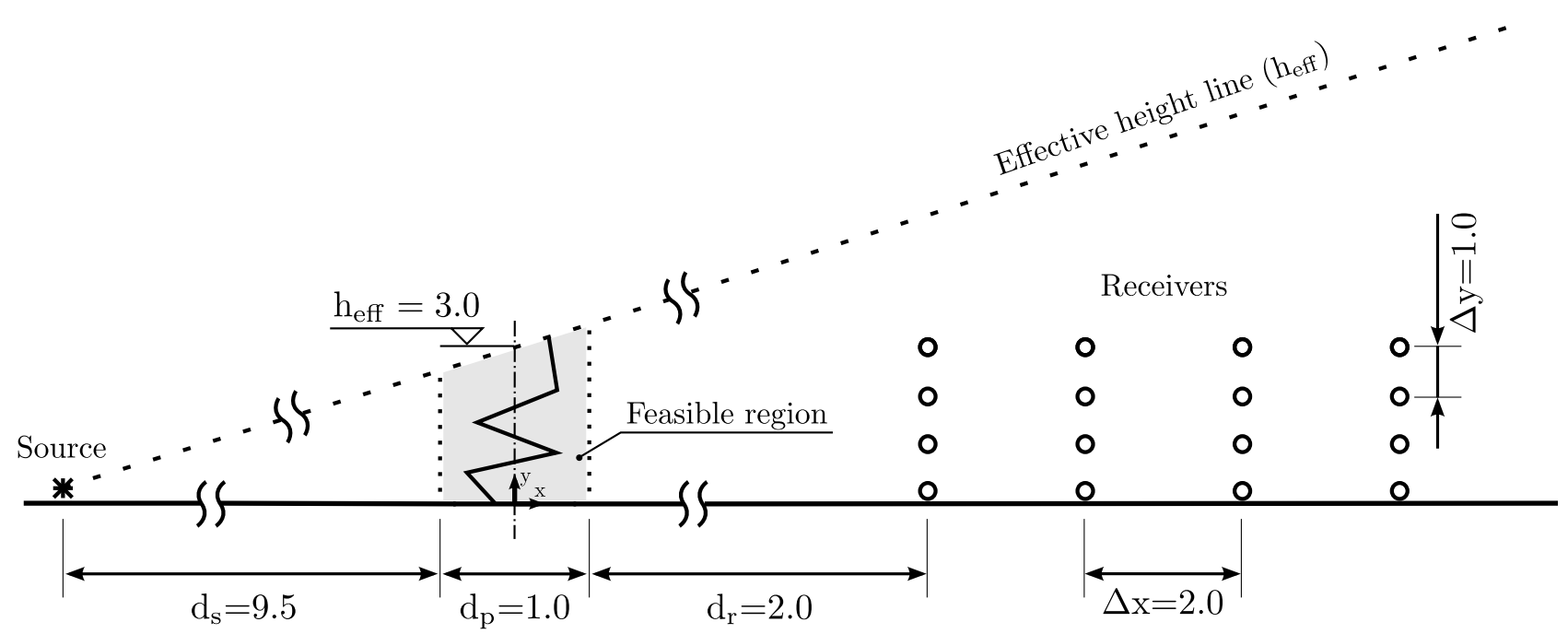

Figure 4: Bi-dimensional configuration to be used in the optimisation process of thin noise barriers. Distances expressed in $[\mathrm{m}]$.

\section{Problem definition}

Fig. 4 represents the general configuration of the model under study. It deals with a two-dimensional model concerning an infinite, coherent mono-frequency source of sound placed on a ground with a perfectly reflecting surface $\left(\beta_{g}=0\right)$ at $\mathrm{d}_{\mathrm{s}}=9.5 \mathrm{~m}$ from the feasible region, parallel to an infinite thin cross-section noise barrier. A trapezoidal section holds the area for feasible profiles, defined by the limited barrier projection to the ground, that is $\mathrm{d}_{\mathrm{p}}=1.0 \mathrm{~m}$, and the maximum effective height to be achieved, that is $\mathrm{h}_{\mathrm{eff}}=3.0 \mathrm{~m}$ at the median of the rectangle trapezium.

A grid of $4 \times 4$ receivers is considered. The first line of receivers lays on the ground and the remaining ones are placed at different heights, vertically separated by $\Delta y=1.0 \mathrm{~m}$. A horizontal distance of $\Delta \mathrm{x}=2.0 \mathrm{~m}$ among them is considered. The nearest receivers to the side limit of the feasible region are $d_{r}=2.0 \mathrm{~m}$ away (see Fig. (4). The proximity of the receivers to the barrier is motivated by the fact that the barrier performance in near regions is more affected by the shape design rather than by the effective height, as occurs in non-near regions.

In the harmonious problem, for every frequency from the analysed noise source, the effectiveness of the barrier design under study is given in terms of the insertion loss (IL), defined as usual:

$$
\mathrm{IL}=-20 \cdot \log _{10}\left(\frac{\mathrm{P}_{\mathrm{B}}}{\mathrm{P}_{\mathrm{HS}}}\right)[\mathrm{dB}]
$$

on every frequency of the band spectrum, and represents the difference of sound pressure levels at the receiver points in the situation with $\left(\mathrm{P}_{\mathrm{B}}\right)$ and without $\left(\mathrm{P}_{\mathrm{HS}}\right)$ considering the barrier.

With the purpose of conducting an optimisation process where the excitation is represented by a noise source pulsing at every frequency of the band spectrum, the efficiency of the barrier for a specific 
receiver can be written as:

$$
\overline{\mathrm{IL}}=-10 \cdot \log _{10}\left(\frac{\sum_{\mathrm{i}=1}^{\mathrm{NF}} 10^{\left(\mathrm{A}_{\mathrm{i}}-\mathrm{IL}_{\mathrm{i}}\right) / 10}}{\sum_{\mathrm{i}=1}^{\mathrm{NF}} 10^{\mathrm{A}_{\mathrm{i}} / 10}}\right)[\mathrm{dBA}]
$$

being $\mathrm{NF}$ the studied spectrum number of frequencies, here $\mathrm{NF}=14, \mathrm{~A}_{\mathrm{i}}$ the spectrum A-weighted noise level and $\mathrm{IL}_{\mathrm{i}}$ the insertion loss value for sources pulsing at every frequency of the spectrum, according to (23). In this work, the noise source has been characterised by using the UNE-EN 17933:1998 25] normalized traffic noise spectrum for third-octave band center frequencies, ranging from 100 to $2,000 \mathrm{~Hz}$, that is the same used by the CTE [26].

Concerning the estimator taken into account along the shape optimisation process, it is worth noting that it is based on the overall IL mean value of all receiver points:

$$
\mathrm{FF}=\sum_{\mathrm{j}=1}^{\mathrm{NR}} \overline{\mathrm{IL}}_{\mathrm{j}} / \mathrm{NR}
$$

being $\overline{\mathrm{IL}}_{\mathrm{j}}$ the IL mean value for each receiver (see (24)) and NR the total number of receivers. This value corresponds to the so called fitness function (FF) to be maximized, according to the proper terminology used in the field of evolutionary algorithms.

\section{Shape optimisation}

Shape design optimisation is carried out by the combined use of an evolutionary algorithm and a code that implements a Dual BEM formulation. The evolutionary algorithm software used in this work applies the GAlib package [27. This library is a collection of $\mathrm{C}++$ genetic algorithm (GA) components from which it is possible to quickly construct GA's to attack a wide variety of problems.

In this paper, for a good equilibrium between exploration and exploitation a steady-state genetic algorithm is used replacing the two worst individuals (in terms of their fitness function) at each generation, with a population size of 100 individuals. A single-point crossover operator is used in this study, with a crossover rate of 0.9 . The considered mutation rate is $1 / n_{c h}$, where $n_{c h}$ is the chromosome length $\left(\mathrm{n}_{\mathrm{ch}}=8 \mathrm{xn}\right.$, being $\mathrm{n}$ the overall number of the design variables -of 8 bits precision each-). Five independent runs of the optimisation process are considered for each model. The stopping criterion condition is met for 20,000 evaluations of the fitness function (FF).

Following [16, 17, a simple procedure to mathematically represent the geometry of barriers is proposed. The design points of the screen model are defined in a systematic, simple way in a reference domain as a previous step to the barrier profile generation in the real space. In short, the transformed domain holds the set of design variables of the model under study, denoted by $\left(\xi_{i}, \eta_{i}\right)$, and represents the rectangular search space for the GA (see left part of Fig. 5 ). Every $\left(\xi_{i}, \eta_{i}\right)$ point in the transformed domain has its image $\left(\mathrm{x}_{\mathrm{i}}, \mathrm{y}_{\mathrm{i}}\right)$ in the Cartesian space, that is the real domain where the barrier operates. 


\section{Transformed Domain}

Cartesian 2D barrier domain

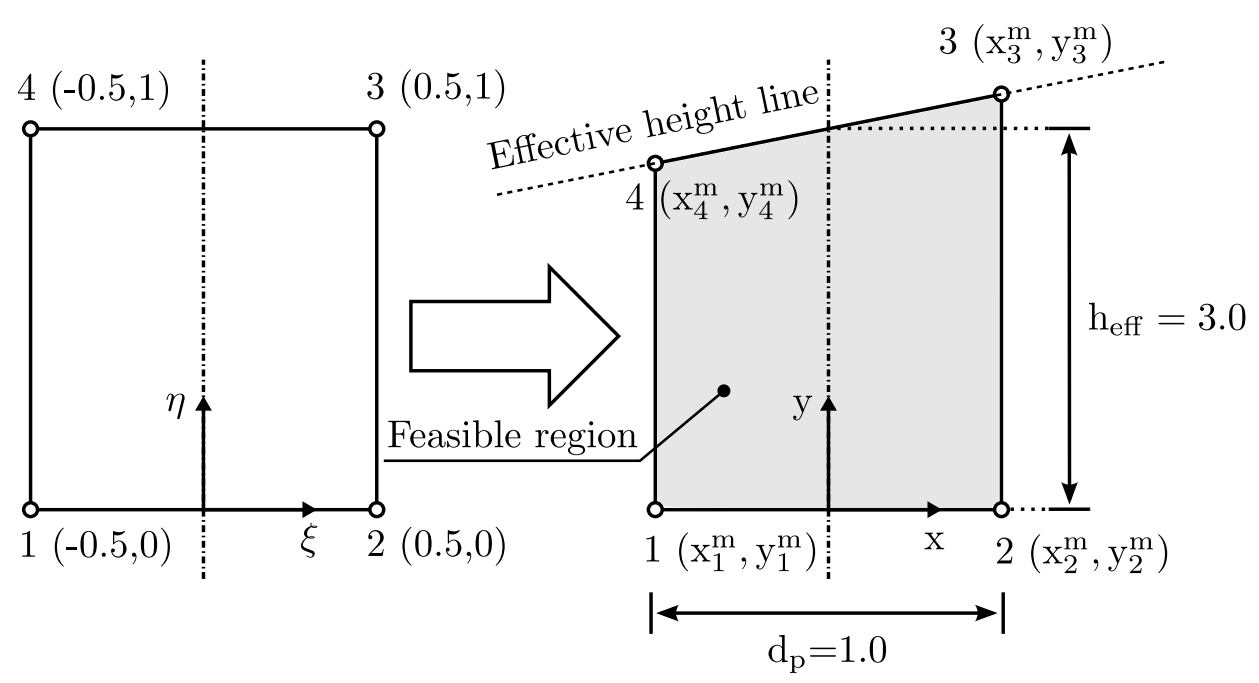

Figure 5: Bi-dimensional coordinate systems. Dimensions expressed in [m].

The transformation of Fig. [5 can be expressed as follows:

$$
\left\{\begin{array}{l}
x_{i} \\
y_{i}
\end{array}\right\}=\gamma_{1}\left\{\begin{array}{l}
x_{1}^{m} \\
y_{1}^{m}
\end{array}\right\}+\gamma_{2}\left\{\begin{array}{l}
x_{2}^{m} \\
y_{2}^{m}
\end{array}\right\}+\gamma_{3}\left\{\begin{array}{l}
x_{3}^{m} \\
y_{3}^{m}
\end{array}\right\}+\gamma_{4}\left\{\begin{array}{l}
x_{4}^{m} \\
y_{4}^{m}
\end{array}\right\}
$$

where:

$$
\begin{aligned}
\gamma_{1}=\left(\frac{1}{2}-\xi\right)(1-\eta) ; \gamma_{2} & =\left(\frac{1}{2}+\xi\right)(1-\eta) \\
\gamma_{3}=\eta\left(\frac{1}{2}+\xi\right) ; \gamma_{4} & =\eta\left(\frac{1}{2}-\xi\right)
\end{aligned}
$$

and:

$$
\begin{gathered}
\mathrm{x}_{1}^{\mathrm{m}}=\mathrm{x}_{4}^{\mathrm{m}}=-\frac{\mathrm{d}_{\mathrm{p}}}{2} ; \mathrm{x}_{2}^{\mathrm{m}}=\mathrm{x}_{3}^{\mathrm{m}}=\frac{\mathrm{d}_{\mathrm{p}}}{2} \\
\mathrm{y}_{1}^{\mathrm{m}}=\mathrm{y}_{2}^{\mathrm{m}}=0 ; \mathrm{y}_{3}^{\mathrm{m}}=\mathrm{h}_{\text {eff }}\left(\frac{\mathrm{d}_{\mathrm{s}}+\mathrm{d}_{\mathrm{p}}}{\mathrm{d}_{\mathrm{s}}+\left(\mathrm{d}_{\mathrm{p}} / 2\right)}\right) ; \mathrm{y}_{4}^{\mathrm{m}}=\mathrm{h}_{\text {eff }}\left(\frac{\mathrm{d}_{\mathrm{s}}}{\mathrm{d}_{\mathrm{s}}+\left(\mathrm{d}_{\mathrm{p}} / 2\right)}\right)
\end{gathered}
$$

In this paper $h_{\text {eff }}=3.0 \mathrm{~m}$ is proposed. This value and the maximum barrier projection to the ground $d_{p}$ have been chosen according to the geometric dimensions of the barriers studied herein and present in the bibliography. Both latter parameters define the feasible region by generating a trapezoidal search space in the Cartesian barrier domain (see right part of Fig. 5 ). Its final dimensions are dependent, logically, of the placement of the noise source $\left(d_{s}\right)$. 


\section{Application of the proposed methodology to the assessment of the acoustic efficiency of different barrier designs}

The proposed methodology previously described is applied on the study of different barrier designs in this section. The diversity of the models chosen in this work has been intended to highlight the robustness and flexibility of the methodology, as well as the wide range of possible geometric designs to be assessed. To expose the scope and features of the method, eight different designs are studied (see Fig. 6), based either on an overall shape or in a top edge optimisation of the barrier model, depending on the case. As for the overall shape design optimisation, five barrier models are proposed: models a) and b) (polygonal-shaped designs), models c) and d) (splines-based models) and model e) (Y-shaped barrier) (see left part of Fig. 6). As for the optimisation of the top edge of the barrier, model f) (tree-shaped barrier), model g) (Y-variant model) and model h) (fork-shaped model) are studied (right part of Fig. 6). Such models are based on a set of points defined by design variables in a transformed domain proposed by the evolutionary algorithm, according to the geometric model definition. It is worth stressing that contrary to the general approach (Fig. 51), the feasible space is constrained to the barrier top in the Cartesian domain for models $f$ ), g) and $h$ ). The authors of this work will refer to the models previously cited as their corresponding names in brackets hereinafter. The considered configurations depicted in Fig. 6) are described in more detail as follows:

\subsection{Polygonal-shaped barrier}

This design is based on a set of points through which straight boundaries are consecutively connected. These points feature a completely free movement inside the feasible region with the exception of the first and the last one, placed on the ground and at the effective height line, respectively.

Two different configurations of this design are studied. The first one is a 3-sided polygonal-shaped barrier (model a)); the second is a 5 -sided polygonal-shaped one (model b)).

The geometry feasibility of the individuals proposed by the evolutionary algorithm is constrained to the condition of non-cut-off points among boundaries.

\subsection{Splines-based-shaped barrier}

A set of spline-based curves is proposed as a barrier model. The points through which the curves pass are proposed by the evolutionary algorithm to generate the cubic segmental interpolation that leads to the desired barrier model. These points feature a completely free movement inside the feasible region with the exception of the first and the last one, placed on the ground and at the effective height line, respectively.

These continuous, differentiable curves are defined as natural cubic splines, meaning that the second derivative is null at both the starting and the ending point of the overall resulting curve.

For the sake of convenience, the splines expressions are written in parametric form. This choice is in the interest of the geometry feasibility of the individuals proposed by the evolutionary algorithm, by ensuring that the tangent vectors to the curve at both sides of the common points between splines have the same sense (see Fig. 7).

The sharp changes in direction that frequently arise when dealing with this sort of curves require some considerations. For both faithfully representing complex configurations and guaranteeing a good 
convergence of numerical integrations, the implementation of mesh-related strategies is needed. These criteria are implemented in the meshing algorithm of the code. In this way, a more refined mesh around critical points of the splines is generated for each frequency to perfectly fit the geometric discretization to the barrier profile. As parabolic elements are used for the boundary discretization, the placement of, at least, one element is considered around critical points (with the central node of such elements located at these points, i.e., at maximums and minimums).

Two different configurations of this design are studied: a 3 and a 5 cubic splines-shaped barrier (model c) and d), respectively).

The geometry feasibility of the designs is also constrained to the condition of non-cut-off points among boundaries, obviously.

\subsection{Y-shaped barrier}

A commonly used Y-shaped barrier is studied (model e)). This model is based on three points (1, 2 and 3) that feature a completely free movement inside the feasible region. Point 0 lays on the ground and is fixed at the median of the feasible region.

\subsection{Tree-shaped barrier}

This design is a tree-shaped model with four arms on its top (model f)). Points from 1 to 4 can be placed anywhere inside the domain. This enables the tilt of the arms to cover a free range of angles.

The barrier model stands on a vertical, fix bar of $2.5 \mathrm{~m}$ height placed on the median of the feasible region from which the four arms are born.

\subsection{Y-variant-shaped barrier}

This model (model h)) can be understood as an evolution of the Y-shaped design (model e)) by adding two branches at each arm of such design. Two of the branches are born from the ending points of the main arms (points 1 and 6) while the remaining ones do it from the middle. The design variables responsible for the inclination of the main arms are constrained to vertical movements $\left(\eta_{1}\right.$ and $\left.\eta_{6}\right)$ through the left- and right-side limits of the feasible region. The barrier model stands on a vertical, fix bar of $2.5 \mathrm{~m}$ height placed on the median of the feasible region.

The geometry feasibility of the model is constrained to both the condition of non-cut-off points among boundaries and the fact that points from 2 to 5 are always in the upper region enclosed by the main arms in the search domain.

\subsection{Fork-shaped barrier}

This model (model h)) represents a barrier with seven vertical branches that are born from a horizontal tray. The distance among branches remains constant $\left(d_{p} / 6\right)$ while their lengths vary throughout the optimisation process. As in the previous cases, this barrier model stands on a vertical bar of 2.5 m height.

In relation to the surface treatment of the barrier models to be analysed, two different cases are proposed. In one of them (Case 1), a shape optimisation considering perfectly rigid boundaries 
$\left(\beta_{\Gamma}=0\right)$ for all models here presented is carried out. As a reference comparing design, Case 1 absorbing deals with the assessment of the acoustic efficiency of the best profiles of models tree-, Y-variant- and fork-shaped from Case 1, on whose top edge boundaries a specific sound absorbing treatment is considered (marked with a grey line in the right part of Fig. (6). The airflow resistivity and the thickness of the absorbing material are $\sigma=30 \mathrm{kPa} \cdot \mathrm{s} / \mathrm{m}^{2}$, which corresponds to mineral wool, and $\mathrm{t}=0.05 \mathrm{~m}$, respectively. In Case 2, a top edge optimisation of barriers tree-, Y-variant- and fork-shaped considering the same surface treatment on crowning boundaries than in Case 1 absorbing is conducted. These research cases aim at determining whether or not absorbing boundary conditions are needed to be considered within the optimisation process, in order to find the best affordable design solutions in terms of acoustic efficiency.

\section{Results and discussion}

Results are shown for the best individuals from the optimisation processes. The left part of the figures (from Fig. 8 11) presented in this section illustrates the optimum designs for each model. The fitness function (FF) and the standard deviation with respect to the IL distribution values of the receivers configuration (SD) are on the upper side of their corresponding barrier profiles. The rightmost graphs show the results concerning the acoustic efficiency of the aforementioned models. One of them displays the average frequency-related IL (from (23)) of the grid of receivers. The other graph shows the average IL spectrum (from (24)) for the set of receivers at the same height. This latter assessment is conducted considering a set of four groups of ten receivers each. The first group lays on the ground and the remaining ones are placed at different heights, vertically separated by $\Delta \mathrm{y}=1.0 \mathrm{~m}$. A horizontal distance of $\Delta \mathrm{x}=1.0 \mathrm{~m}$ among them is considered, being the nearest ones at $\mathrm{d}_{\mathrm{r}}=0.5 \mathrm{~m}$ from the feasible region.

Figures 8, 9 and 10 show the results concerning the optimisation of the models under the consideration of perfectly rigid boundaries (Case 1). Figure 11 shows the results concerning the top edge optimisation of models featuring boundaries with absorbing surface (Case 2).

Table 1 shows the acoustic efficiency gain of the models with respect to a $3-\mathrm{m}$ height straight barrier with rigid boundaries. Table 2 collects the coordinates in the transformed domain of the design variables of the best individual found along the optimisation runs for each barrier model.

Table 1: Acoustic efficiency of the models under study, in [dBA].

\begin{tabular}{lcccccc}
\hline \multirow{2}{*}{ Model } & \multicolumn{3}{c}{ Case 1 } & Case 1 & absorbing & \multicolumn{2}{c}{ Case 2 } \\
\cline { 2 - 7 } & $\mathrm{FF}$ & $\Delta \mathrm{FF}^{*}$ & $\mathrm{FF}$ & $\Delta \mathrm{FF}^{*}$ & $\mathrm{FF}$ & $\Delta F^{*}$ \\
\hline a) 3-sided polygonal & 19.27 & +4.73 & - & - & - & - \\
b) 5-sided polygonal & 20.54 & +6.00 & - & - & - & - \\
c) 3-cubic splines & 19.03 & +4.49 & - & - & - & - \\
d) 5-cubic splines & 19.32 & +4.78 & - & - & - & - \\
e) Y-shaped & 19.29 & +4.75 & - & - & - & - \\
f) Tree-shaped & 20.52 & +5.98 & 20.94 & +6.40 & 21.41 & +6.87 \\
g) Y-variant-shaped & 21.29 & +6.75 & 21.13 & +6.59 & 22.00 & +7.46 \\
h) Fork-shaped & 21.20 & +6.66 & 20.82 & +6.28 & 21.78 & +7.24 \\
\hline$* \Delta \mathrm{FF}=\mathrm{FF}_{\text {Model }}-\mathrm{FF}_{\text {Straight Rigid Barrier }}$ & & & & & &
\end{tabular}




\section{OVERALL SHAPE DESIGN OPTIMIZATION}

Reference Point in

Transformed Domain

a)

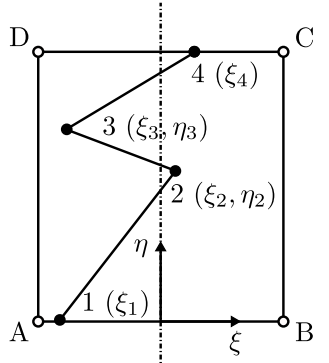

Genome

\begin{tabular}{|l|l|l|l|l|l|}
\hline$\xi_{1}\left|\xi_{2}\right|$ & $\eta_{2}$ & $\xi_{3}$ & $\eta_{3}$ & $\xi_{4}$ \\
\hline
\end{tabular}

b)

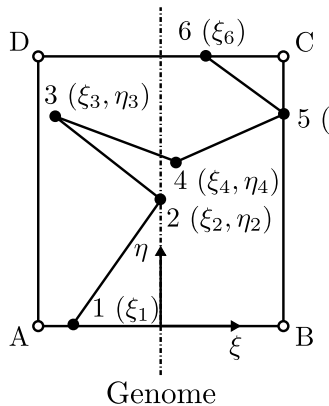

$\xi_{1}\left|\xi_{2}\right| \eta_{2}\left|\xi_{3}\right| \eta_{3}\left|\xi_{4}\right| \eta_{4}\left|\xi_{5}\right| \eta_{5} \mid \xi_{6}$

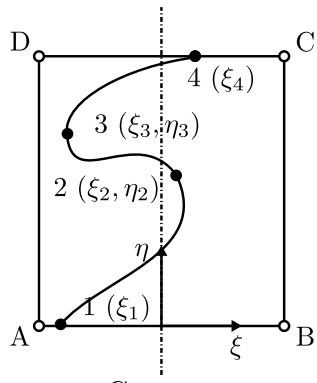

Genome

\begin{tabular}{|l|l|l|l|l|l|l|}
$\xi_{1}$ & $\xi_{2}$ & $\eta_{2}$ & $\xi_{3}$ & $\eta_{3}$ & $\xi_{4}$ \\
\hline
\end{tabular}

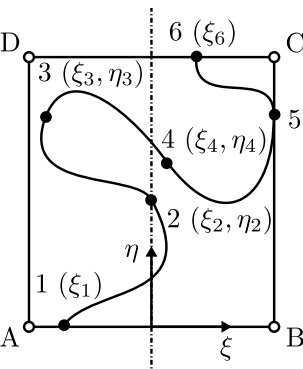

Genome

$\xi_{1}\left|\xi_{2}\right| \eta_{2}\left|\xi_{3}\right| \eta_{3}\left|\xi_{4}\right| \eta_{4}\left|\xi_{5}\right| \eta_{5} \mid \xi_{6}$

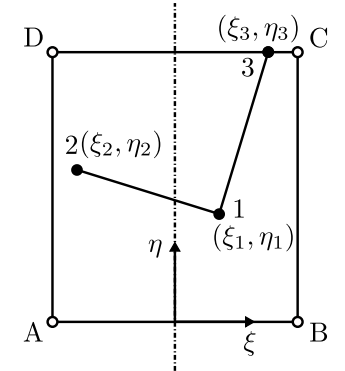

Genome

$\xi_{1}\left|\eta_{1}\right| \xi_{2}\left|\eta_{2}\right| \xi_{3} \mid \eta_{3}$

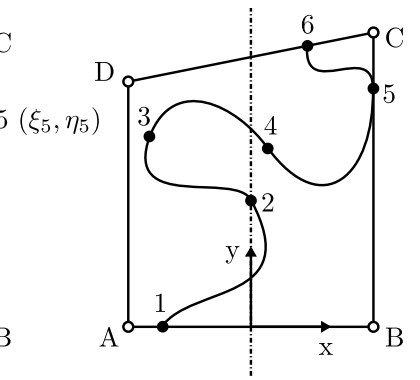

Barrier Profile analyzed in 2D Cartesian Domain

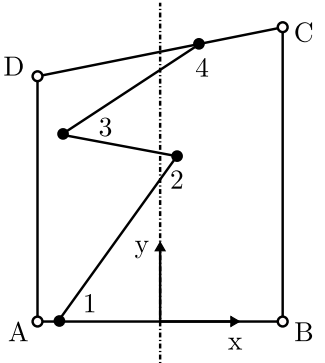

f)

Reference Point in

Transformed Domain

Barrier Profile analyzed

in 2D Cartesian Domain

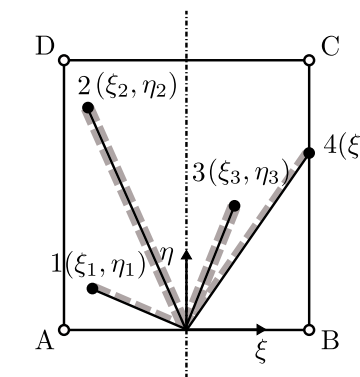

Genome

$\xi_{1}\left|\eta_{1}\right| \xi_{2}\left|\eta_{2}\right| \xi_{3}\left|\eta_{3}\right| \xi_{4} \mid \eta_{4}$
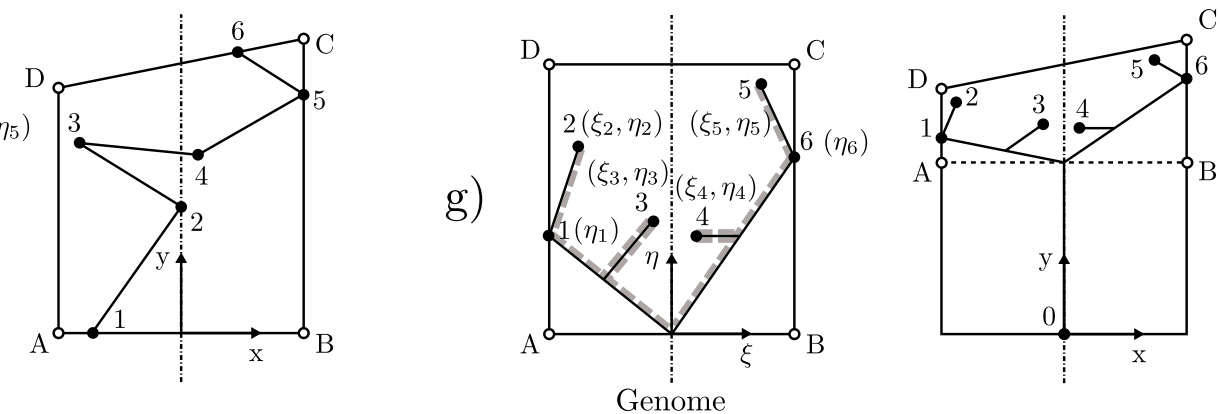

$\eta_{1}\left|\xi_{2}\right| \eta_{2}\left|\xi_{3}\right| \eta_{3}\left|\xi_{4}\right| \eta_{4}\left|\xi_{5}\right| \eta_{5} \mid \eta_{6}$

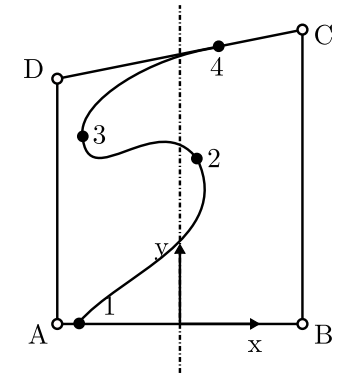

h)
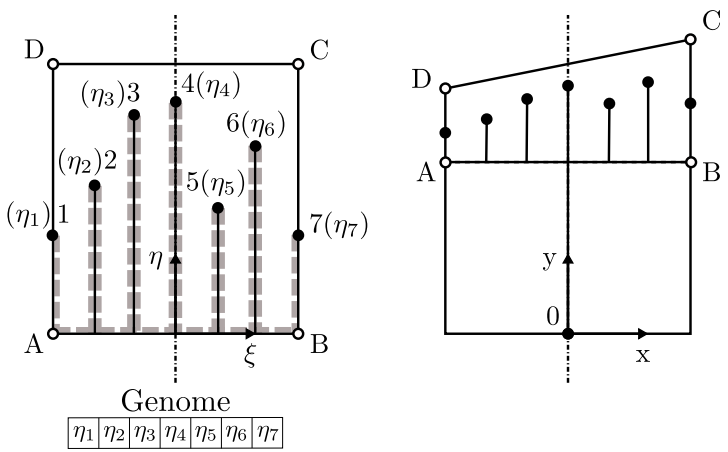

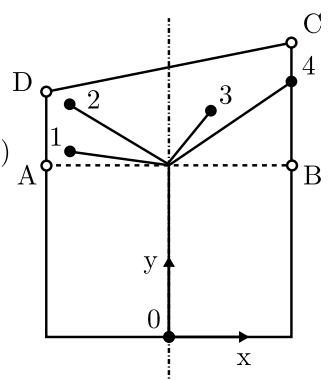

g)

\begin{tabular}{|l|l|l|l|l|l|l|}
$\eta_{1} \mid$ & $\eta_{2}$ & $\eta_{3}$ & $\eta_{4}$ & $\eta_{5}$ & $\eta_{6}$ & $\eta_{7}$ \\
\hline
\end{tabular}

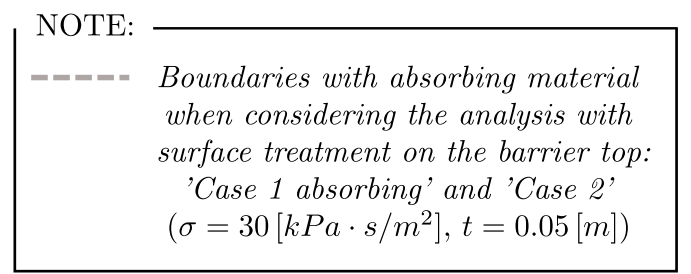

Figure 6: Barrier models studied. 

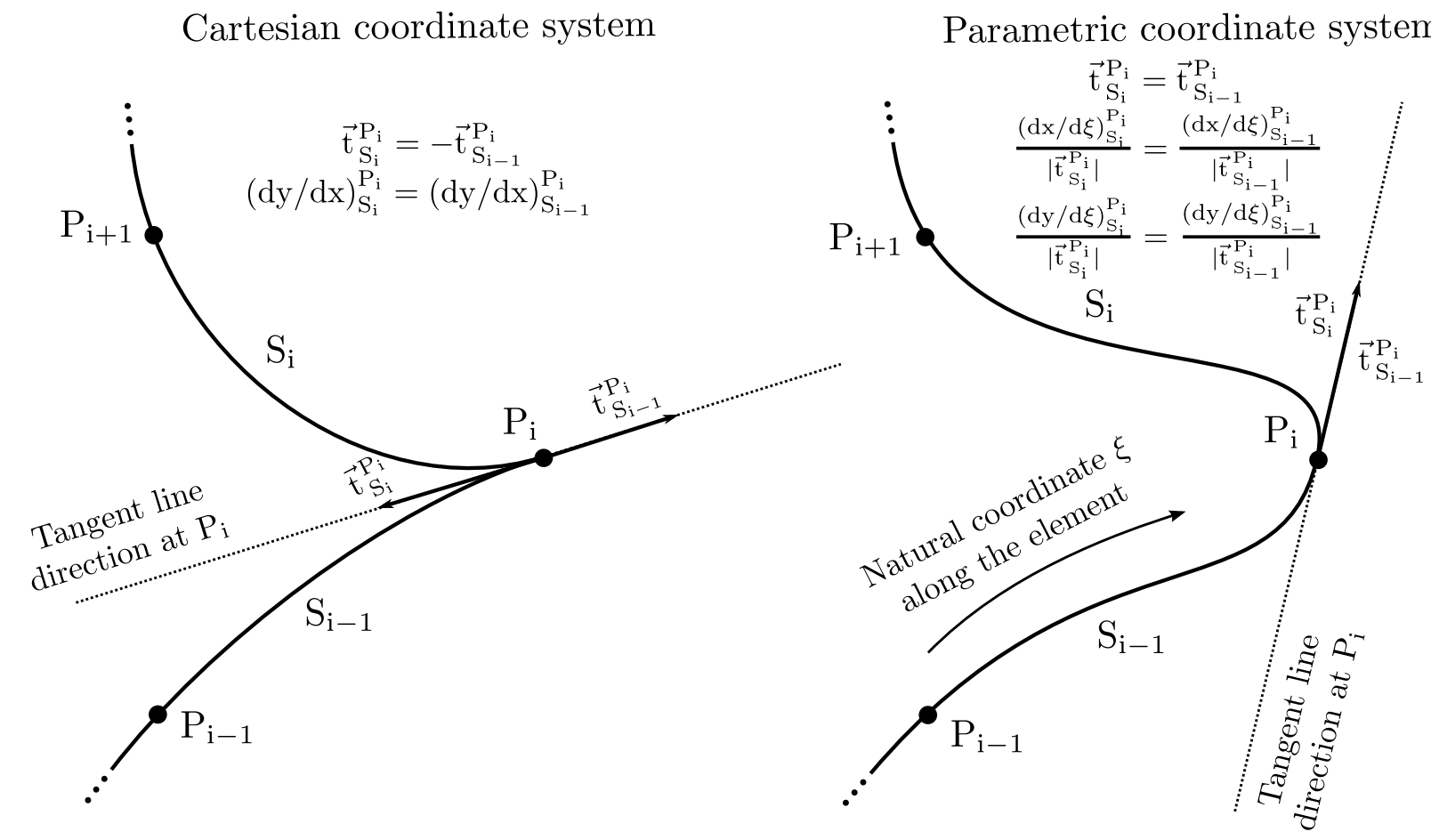

Figure 7: Convenience of the choice of a parametric representation to generate a multiple splinesbased curve. 


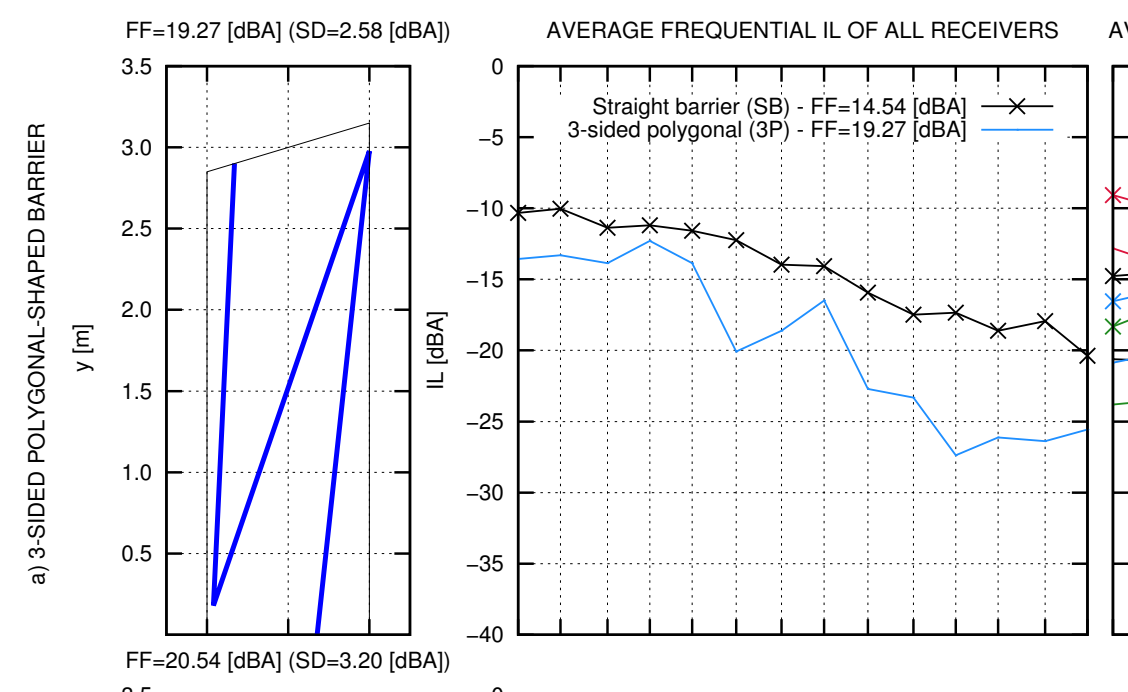

AVERAGE IL SPECTRUM ALONG RECEIVERS' HEIGHT
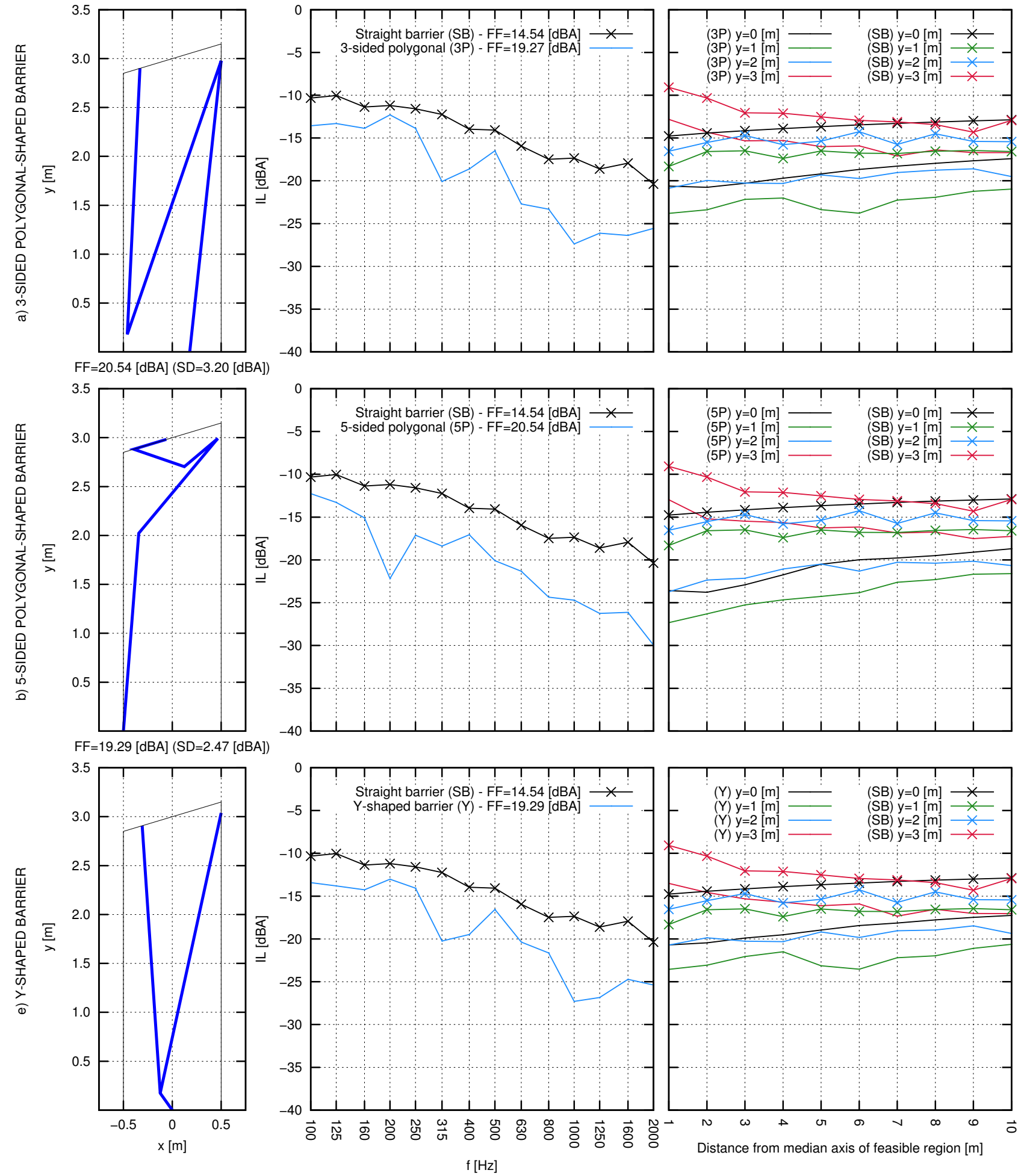

Figure 8: Overall shape design optimisation for polygonal- and $Y$-shaped models (model a), b) and e), respectively). 'Case 1' (rigid boundaries). 


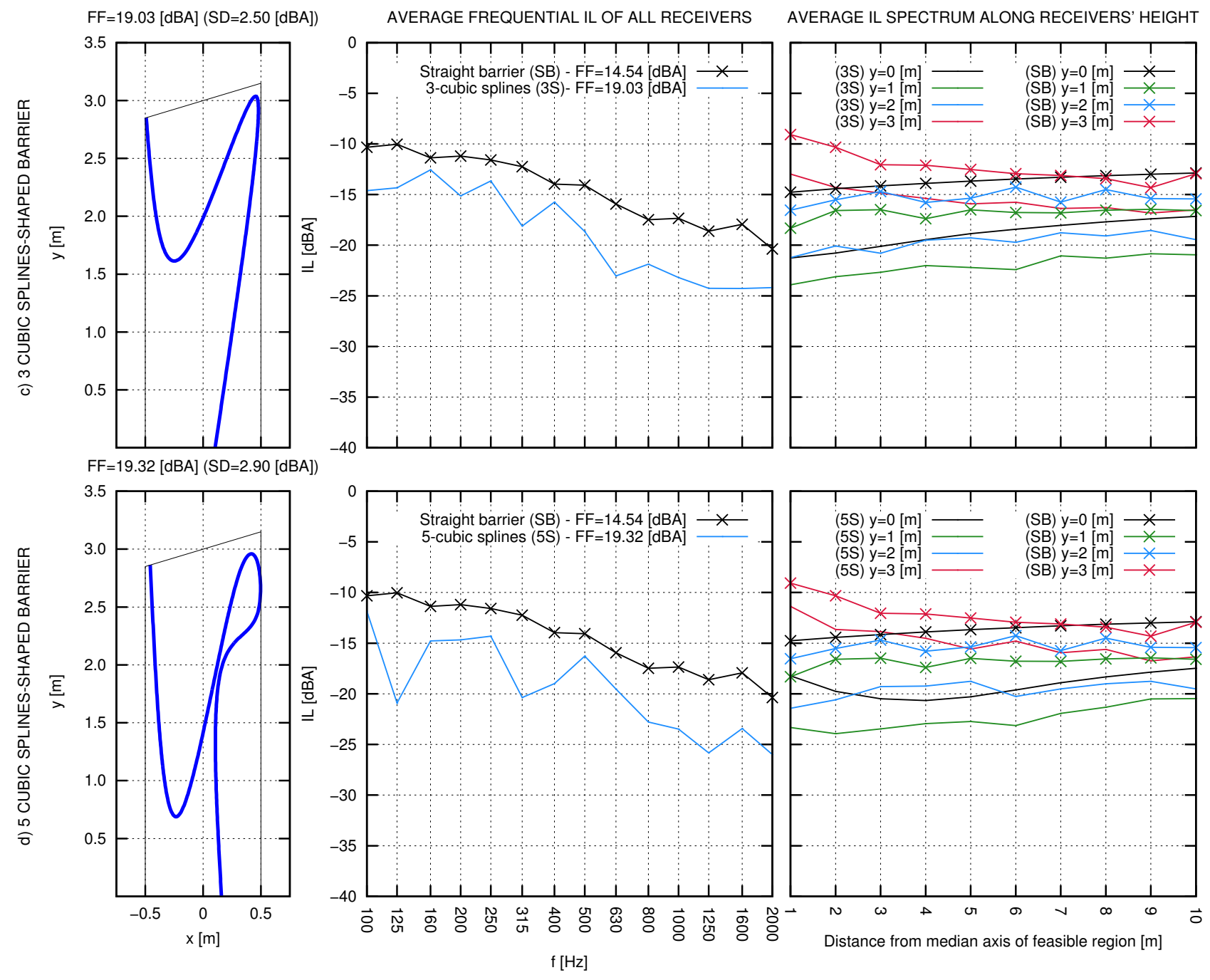

Figure 9: Overall shape design optimisation for multiple cubic splines-based models (model c) and d)). 'Case 1' (rigid boundaries). 

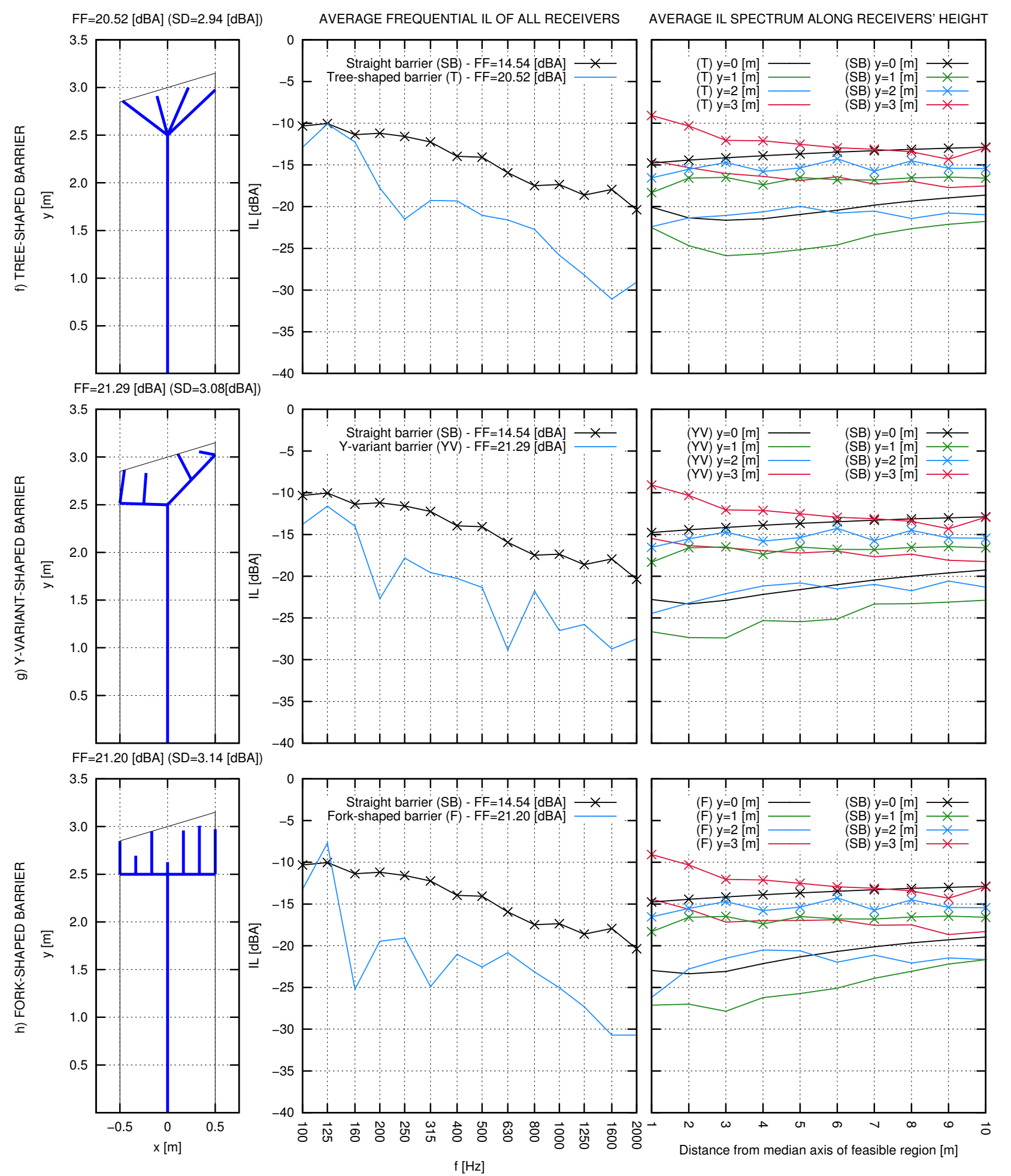

Figure 10: Top edge optimisation for f) tree-, g) Y-variant- and h) fork-shaped model. 'Case 1' (rigid boundaries). 


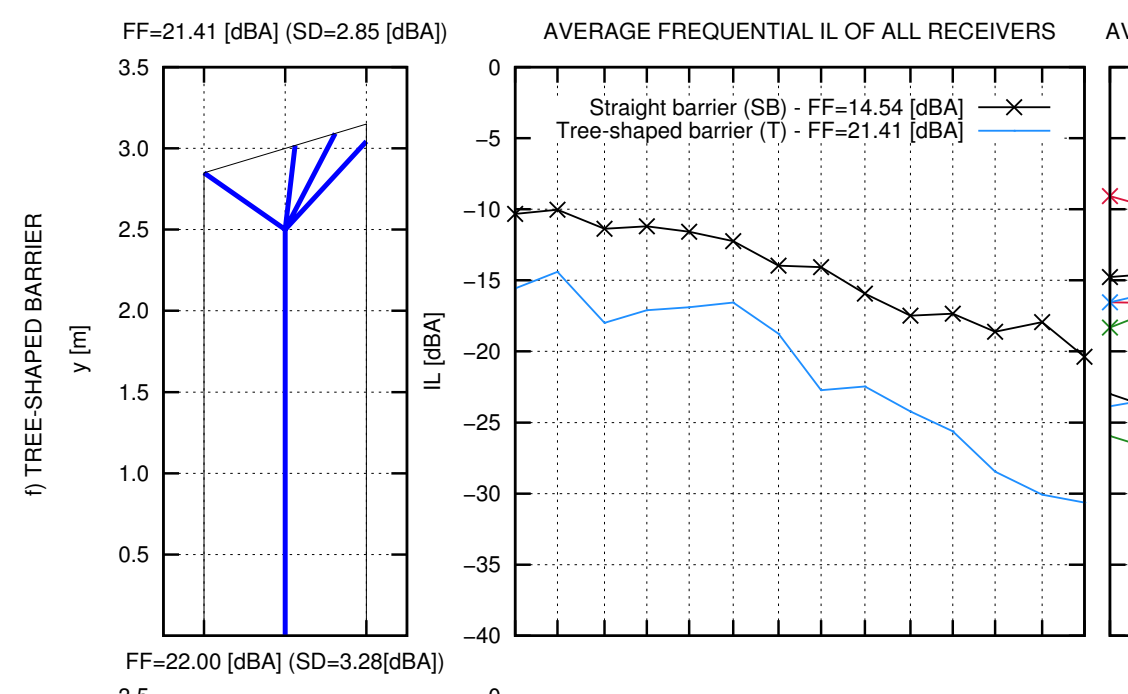

AVERAGE IL SPECTRUM ALONG RECEIVERS' HEIGHT
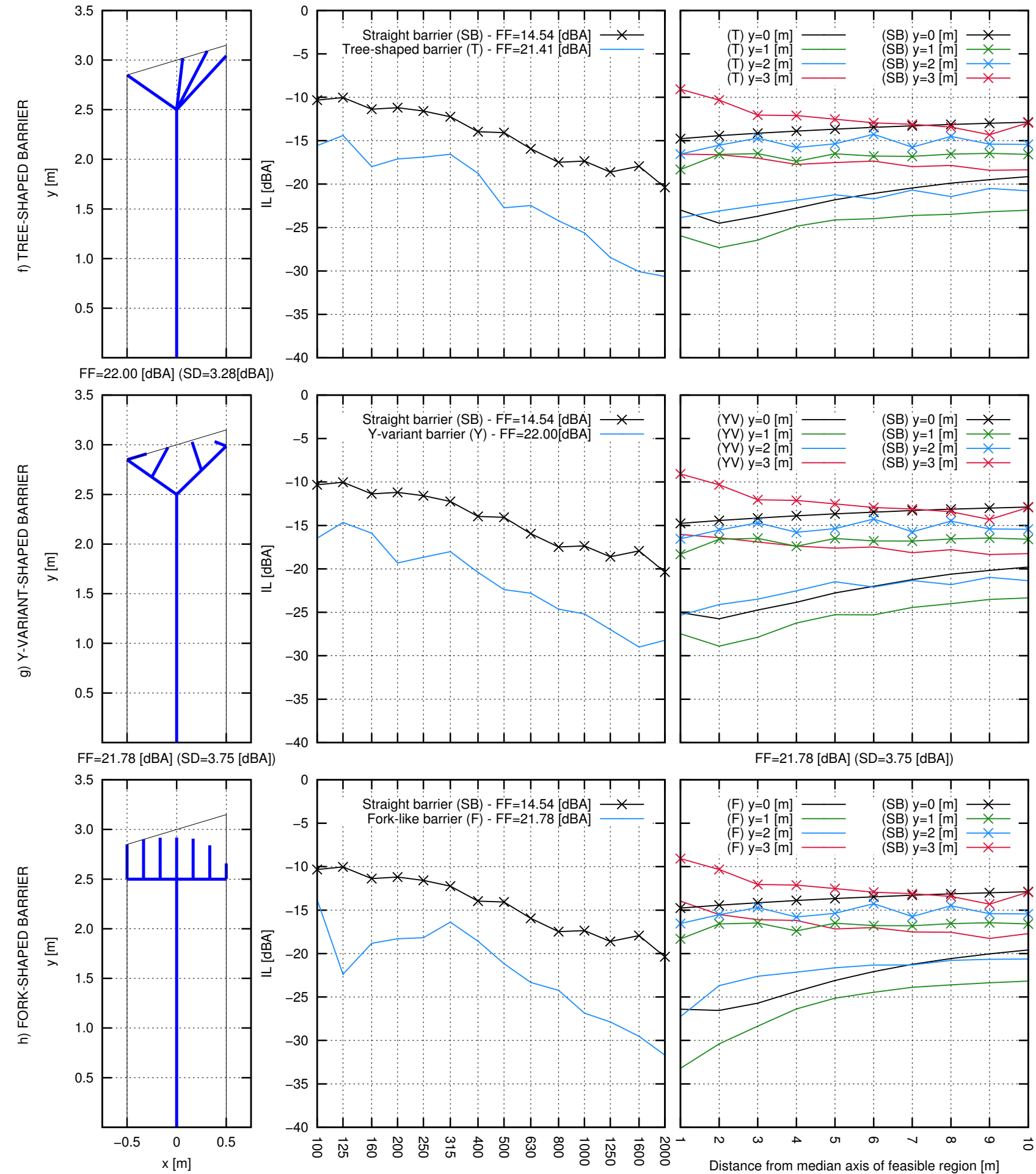

$\mathrm{FF}=21.78[\mathrm{dBA}](\mathrm{SD}=3.75[\mathrm{dBA}])$

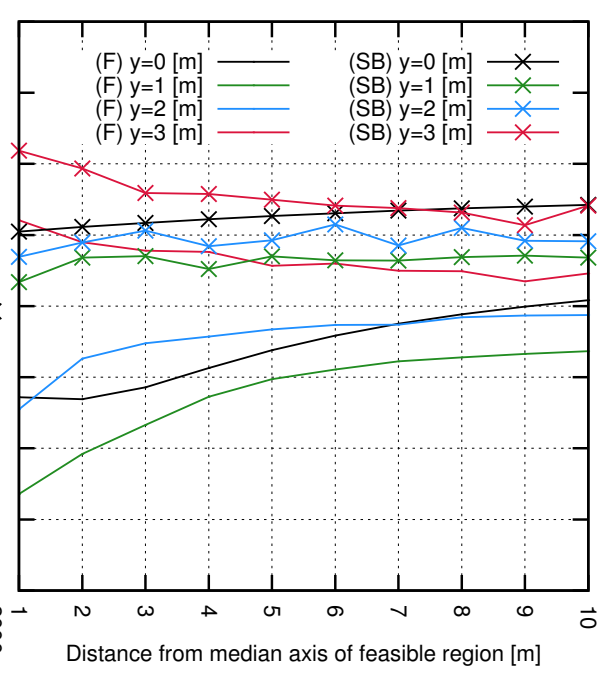

Figure 11: Top edge optimisation for f) tree-, g) Y-variant- and h) fork-shaped model. 'Case 2' (absorbing boundaries). 
Table 2: Design variables in the transformed domain of the best individual found along all optimisation runs for each barrier model.

\begin{tabular}{|c|c|c|c|c|c|c|c|c|c|c|c|c|c|c|}
\hline Case & Model & $\xi_{1}$ & $\eta_{1}$ & $\xi_{2}$ & $\eta_{2}$ & $\xi_{3}$ & $\eta_{3}$ & $\xi_{4}$ & $\eta_{4}$ & $\xi_{5}$ & $\eta_{5}$ & $\xi_{6}$ & $\eta_{6}$ & $\eta_{7}$ \\
\hline \multirow{8}{*}{1} & a) 3-sided $p$ & 0.17843 & - & 50000 & 0.94510 & -0.46078 & 0.06275 & -0.33137 & - & - & - & - & - & - \\
\hline & b) 5-sided polygonal & -0.50000 & - & -0.34314 & 0.69804 & 0.46471 & 0.95294 & 0.12353 & 0.89020 & -0.39412 & 1.00000 & -0.06078 & - & - \\
\hline & c) 3-cubic splines & 0.10392 & - & 0.46471 & 0.96471 & -0.17059 & 0.56471 & -0.49216 & - & - & - & - & - & - \\
\hline & d) 5-cubic splines & 0.15882 & - & 0.14706 & 0.63137 & 0.47647 & 0.78431 & 0.30784 & 0.89804 & -0.20980 & 0.23922 & -0.45686 & - & - \\
\hline & e) Y-shaped & -0.12353 & 0.05882 & -0.30784 & 1.00000 & 0.50000 & 0.96471 & - & - & - & - & - & - & - \\
\hline & f) Tree-shaped & -0.11177 & 0.87843 & -0.47255 & 1.00000 & 0.50000 & 0.72941 & 0.21765 & 0.88235 & - & - & - & - & \\
\hline & g) Y-variant-shaped & - & 0.04314 & -0.45324 & 1.00000 & -0.22500 & 0.76863 & 0.10814 & 1.00000 & 0.33147 & 0.92549 & - & 0.80392 & - \\
\hline & h) Fork-shaped & - & 0.98824 & - & 0.48628 & - & 1.00000 & - & 0.25490 & - & 0.83529 & - & 0.84706 & 0.72549 \\
\hline \multirow{3}{*}{2} & f) Tree-shaped & 0.30784 & 1.00000 & -0.50000 & 1.00000 & 0.50000 & 0.83529 & 0.06078 & 1.00000 & & & & & \\
\hline & g) Y-variant-shaped & - & 1.00000 & -0.30235 & 1.00000 & -0.08775 & 1.00000 & 0.15520 & 0.96863 & 0.38265 & 0.86667 & 0.74902 & - & \\
\hline & h) Fork-shaped & - & 1.00000 & - & 1.00000 & - & 0.92549 & - & 0.83529 & - & 0.74118 & - & 0.56863 & 0.24314 \\
\hline
\end{tabular}


From the analysis of the results obtained some conclusions on the response of the models studied and, most importantly, on the procedure described in this work may be drawn:

- In line with other authors ([4], [6], [7], 8], [28]), acting on the top of the barrier is found to be an appropriate strategy to minimize the acoustic impact. This is illustrated by the fact that, on the whole, models based on their top edge optimisation feature a better acoustic performance than those whose overall shape has been optimised. Furthermore, the latter models equally display a tendency towards the modification of their top edge in the search for the best acoustic performance.

- The barrier performance when applying sound-absorbing materials to some boundaries of its optimised reflecting profile (Case 1 absorbing) may lead to unexpected results, as the configuration of the top of sound reflecting barriers plays an important role by producing reflected waves that help partly offset the incident ones. The incidence of this effect largely depends on the crowning configuration of the models studied here, ranging from a gain of $0.4 \mathrm{dBA}$ for the tree-shaped barrier (model f)) to a loss of the same value for the fork-shaped barrier (model h)) when compared to their respective performance for rigid boundaries condition (Case 1).

- Considering absorbing boundaries condition within the optimisation process (Case 2) is necessary to give assurance that the search leads to the best affordable profiles in terms of acoustic efficiency. This is supported by the fact that the performance of the best individuals from the top edge optimisation of f) tree-, g) Y-variant- and h) fork-shaped barrier under this consideration, clearly outperforms the acoustic efficiency of such models from Case 1 absorbing (between 0.5 and $1.0 \mathrm{dBA}$ ).

- The average IL spectrum values tend to remain roughly regular with the receiver distance to the barrier for the range studied. The fork-shaped barrier (model h)) shows a far better acoustic behaviour for close receiver points (between 5 and $10 \mathrm{dBA}$ ), though.

\section{Conclusions}

A methodology to successfully optimize thin noise barriers by idealizing their profiles as null crosssection boundaries has been presented. With the purpose of highlighting the robustness, flexibility and the wide range of possibilities of the method, some template configurations have been analysed in this work, ranging from complex straight boundary configurations to curve-shaped profiles, from overall shape designs to top edge configurations. Nevertheless, this methodology may be applied to any real geometric thin design with immediate practical application for its performance to be improved.

The versatility of the algorithm responsible for the geometry generation of the barrier makes the building of the profile to be easily accomplished. The Dual Boundary Element formulation here presented allows a simple treatment of the geometric shape of thin complex barriers. This is a significant advantage over the case when dealing with geometries of real barrier profiles, as the evaluation process for the feasibility of the design proposed by an evolutionary algorithm is often cumbersome and difficult to establish. To the auhtors' knowledge, the procedure described in this work is the first joint implementation of evolutionary algorithms and a Dual BEM formulation concerning this issue. 
The procedure presented is a useful method to assess the acoustic behaviour of thin complex noise barriers configurations and yields conclusions that might have been hardly drawn without its implementation.

\section{Acknowledgments}

This work was supported by the Subdirección General de Proyectos de Investigación of the Ministerio de Economía y Competitividad (MINECO) of Spain and FEDER through research project BIA2010-21399-C02-01 and also by the Agencia Canaria de Investigación, Innovación y Sociedad de la Información (ACIISI) of the Government of the Canary Islands and FEDER through research project ProID20100224. R. Toledo is a recipient of a fellowship from the Subprogram of Predoctoral Fellowships of Research Personnel in Trainning (FPI-MICINN), granted by the Ministerio de Ciencia e Innovación of Spain. The authors are grateful for this support.

\section{References}

\section{References}

[1] S. Seznec. Diffraction of sound barriers: use of the Boundary Element technique. Journal of Sound and Vibration, Vol. 73, 195-209, 1980.

[2] D. Hothersall, S. Chandler-Wilde and M. Hajmirzae. Efficiency of single noise barriers, Journal of Sound and Vibration, Vol. 146, 303-322, 1991.

[3] D. Hothersall, D. Crombie and S. Chandler-Wilde. The performance of T-profile and associated noise barriers. Applied Acoustics, Vol. 32, 269-287, 1991.

[4] G. Watts and P. Morgan. Acoustic performance of an interference type noise- barrier profile. Applied Acoustics, Vol. 49(1), 1-16, 1996.

[5] D. Crombie, D. Hothersall and S. Chandler-Wilde. Multiple-edge noise barriers. Applied Acoustics, Vol. 44, 353-367, 1995.

[6] M. Monazzam and Y. Lam. Performance of profiled single noise barriers co- vered with quadratic residue diffusers. Applied Acoustics, Vol. 66, 709-730, 2005.

[7] Ishizuka and K. Fujiwara. Performance of noise barriers with various edge shapes and acoustical conditions. Applied Acoustics, Vol. 65, 125-141, 2004.

[8] Okubo and K. Fujiwara. Efficiency of a noise barrier on the ground with an acoustically soft cylindrical edge. Journal of Sound and Vibration, Vol. 216(5), 771-790, 1998.

[9] P. Jean, J. Defrance and Y. Gabillet. The importance of source type on the assessment of noise barriers. Journal of Sound and Vibration, Vol. 226(2), 201-206, 1999.

[10] O. Maeso and J. J. Aznárez. Estrategias para la reducción del impacto acústico en el entorno de carreteras. Una aplicación del Método de los Elementos de Contorno. Universidad de Las Palmas de Gran Canaria. ISBN: 84-689- 0340-X, http://hdl.handle.net/10553/1500, doi:846890340X, 2005. 
[11] A. Tadeu, J. António, L. Godinho and P. A. Mendes. Simulation of sound absorption in 2D thin elements using a coupled BEM/TBEM formulation in the presence of fixed and moving 3D source. Journal of Sound and Vibration, Vol. 331, 2386-2403, 2012.

[12] L. de Lacerda, L. Wrobel and W. Mansur. A dual boundary element formulation for sound propagation around barriers over an impedance plane. Journal of Sound and Vibration, Vol. 202(2), 235-247, 1997.

[13] I. Chen, J. Lee, Y. Hsiao and J. Chen. On physical and numerical resonances for water wave problems using the dual boundary element method. Engineering Analysis with Boundary Elements, Vol. 36, 1571-1580, 2012.

[14] D. Duhamel. Shape optimization of noise barriers using genetic algorithms. Journal of Sound and Vibration, Vol. 297, 432-443, 2006.

[15] M. Baulac, J. Defrance and P. Jean. Optimisation with genetic algorithm of the acoustic performance of T-shaped noise barriers with a reactive top surface. Applied Acoustics, Vol. 69, 332-342, 2006.

[16] D. Greiner, J. J. Aznárez, O. Maeso and G. Winter. Shape design of noise barriers using Evolutionary optimisation and Boundary Elements. The Fifth International Conference on Engineering Computacional Technology, Civil-Comp-Press, Stirlingshire, UK: Civil-Comp Press, Vol. 43, 2006.

[17] D. Greiner, J. J. Aznárez, O. Maeso and G. Winter. Single- and multi-objective shape design of Ynoise barriers using Evolutionary computation and Boundary Elements. Advances in Engineering Software, Elsevier, Vol. 41, 368-378, 2010.

[18] D. Greiner, B. Galván, J. J. Aznárez, O. Maeso and G. Winter. Robust design of noise attenuation barriers with Evolutionary multiobjective algorithms and the Boundary Element Method. NCS, Evolutionary Multi-Criterion optimisation, Eds: M. Ehrgott et al., Springer, Vol. 5467, 261-274, 2009.

[19] S. Grubeša, K. Jambrošić and H. Domitrović. Noise barriers with varying cross-section optimized by genetic algorithms. Applied Acoustics, Vol. 73, 1129-1137, 2012.

[20] K. Deb, S. Bandaru, D. Greiner, A. Gaspar-Cunha and C. Celal Tutum. An integrated approach to automated innovization for discovering useful design principles: Case studies from engineering. Applied Soft Computing, Vol. 15, 42-56, 2014.

[21] A. Sáez, R. Gallego and J. Domínguez. Hypersingular quarter-point boundary elements for crack problems. International Journal for Numerical Methods in Engineering, Vol. 38, 1681-1701, 1995.

[22] O. von Estorff (Ed.). Boundary Elements in Acoustics. Advances \& Applications, 2000.

[23] M. Delany and E. Bazley. Acoustical properties of fibrous absorbent materials. Applied Acoustics, Vol. 3, 105-116, 1970.

[24] J. Telles. A self-adaptative co-ordinate transformation for efficient numerical evaluation of general boundary element integrals. International Journal for Numerical Methods in Engineering, Vol. 24, 959-973, 1987. 
[25] UNE-EN 1793-3:1998 - Road traffic noise reduction devices. Test method for determining the acoustic performance. Part 3: Normalized traffic noise spectrum, 1997.

[26] Código Técnico de la Edificación. Documento Básico HR. Protección frente al ruido. Espectro normalizado del ruido de automóviles, ponderado A (Tabla A.3). Ministerio de Fomento de España, Septiembre, 2009.

[27] M. Wall (Ed.). GAlib: A C ++ Library of Genetic Algorithm Components (http://lancet.it.edu/ga/). Mechanical Engineering Department, Massachusets Institute of Technology, 1996.

[28] M. Baulac, J. Defrance and P. Jean. Optimisation with genetic algorithm of the acoustic performance of T-shaped noise barriers with a reactive top surface. Applied Acoustics, Vol. 69, 332-342, 2008. 